\title{
Based on the Hardware Resources Configurable Shanke PLC Building Energy Consumption Detection System
}

\author{
Guanghe Cheng ${ }^{1,2}$, Shuai Zhao ${ }^{1, a}$, Maoli Wang' ${ }^{2}$ Rui-rui Sun ${ }^{2}$, Xiang Sun ${ }^{2}$ \\ ${ }^{1}$ Qilu University of Technology, 250353,Jinan, Shandong China \\ ${ }^{2}$ Shandong Computer Science Center(National Supercomputer Center in Jinan), Shandong Provincial Key Laboratory \\ of Computer Networks,250014,Jinan,Shandong,China
}

\begin{abstract}
According to the actual situation of the comprehensive office building and the functional requirements of the building energy consumption monitoring and management system, the office building energy consumption monitoring and management system is designed by using the hardware resource configurable Shanke PLC(SKPLC) as the data collector. The system uses data bus technology and field data acquisition technology to achieve the building energy consumption data acquisition and management. Practice has proved that energy-saving effect is good.
\end{abstract}

\section{Introduction}

At present, in China's large public buildings, excessive power consumption is a prominent issue in building energy consumption. According to statistics, public building energy consumption of 63.35 million tons of standard coal, accounting for $3.6 \%$ of the total energy consumption. The energy cost of spending growth rate is much higher than the national energy consumption growth rate. Unit area power consumption is 5 times to the ordinary public buildings, while it is Europe, Japan and other developed countries similar to the building 1.5 to 2 times. Therefore, researching a set of practical building energy consumption monitoring and management system for real-time monitoring and analysis of energy consumption data statistics, it is necessary for the relevant departments to provide energy efficiency assessment basis.

Based on the actual situation of the office building of the eastern district of Shandong Academy of Sciences and the technical characteristics of SKPLC whose hardware resources can be configured. This paper designs a set of monitoring and management system of office building energy consumption based on data bus technology and field data acquisition technology ${ }^{[1]}$. One of the main equipment data acquisition use the Shandong province Computing Center independently developed hardware resources configurable $\mathrm{PLC}^{[4-5]}$. The system mainly completes the classification of

${ }^{\mathrm{a} C o r r e s p o n d i n g ~ a u t h o r: 760825602 @ q . q q . c o m}$ 
large-scale buildings, sub-energy data collection and processing, to achieve the comprehensive office building energy consumption dynamic monitoring and management.

\section{System Composition}

The system includes three types of hardware devices:metering terminal, data acquisition control terminal and data collector; two software parts:data transfer station and data center, web information distribution system. The system architecture is shown in Figure 1.

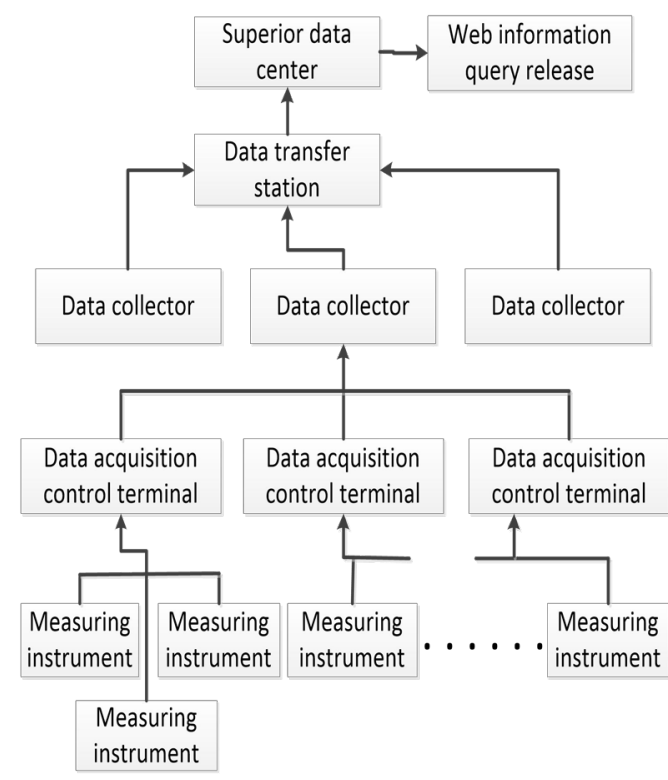

Figure 1. Building energy consumption monitoring and management system architecture

The metering instrument is responsible for energy, water, gas and other classifications for energy consumption data measurement. The data acquisition and control terminal converts the collected meter data into a digital signal. The data collector collects data from the RS485 bus network to collect and control the digital signals of the terminals, and the data acquisition and control terminal controls sends data to the data center or transit station by the LAN and TCP/IP protocol. After data processing, the data center is provided to the web information publishing system for users to access and query the building energy consumption monitoring data through the LAN and browser. In this system, a total of 40 power metering terminals, 6 water flow meters and 8 data collectors were installed.

The data collector is installed in the data acquisition and control terminal centralized location, mainly to complete the acquisition of different types of energy consumption to collect terminal equipment and sub-energy equipment data and upload data to the host computer such as data center server, which is the most complex in this system of the hardware equipment. In the integrated office building building energy consumption monitoring and management system, we use the SKPLC that the hardware resources can be configured as the core of the mountain equipment to set up a data acquisition device.

\section{Realization of Data Acquisition Device of SKPLC Based on Hardware Resource Configuration}

In this system, the data collector collects data of 6 remote water flow meters, 40 power station capable of measuring terminals and 2 central air conditioning equipment, etc., and uploads data to the data center server through the LAN. The hardware resource configurable SKPLC, which is independently developed by Shandong provincial computer center, targets the Siemens S7300 series ${ }^{[2-3]}$, whose hardware resources can be configured, supporting RS85, RS232 and Ethernet interface, having the characteristics of the large capacity I/O channel expansion, modular design, simple programming, reliable performance. After the logic programming of SKPLC, the function of data collector is realized, which is the core equipment of building energy consumption monitoring management system.

\subsection{Power metering terminal data acquisition}


Because the power metering terminal uses the RS485 custom agreement, which is same as the data collector and data center protocol, so the data collector sends the data acquisition command from the data center, directly from the RJ45 forward to connect multiple metering terminal on the RS485 bus, the arrival of a receiving electricity metering terminal in the RS485 interface, Ethernet can be forwarded to RS485. Collect the metering terminal program flow in Figure 2.

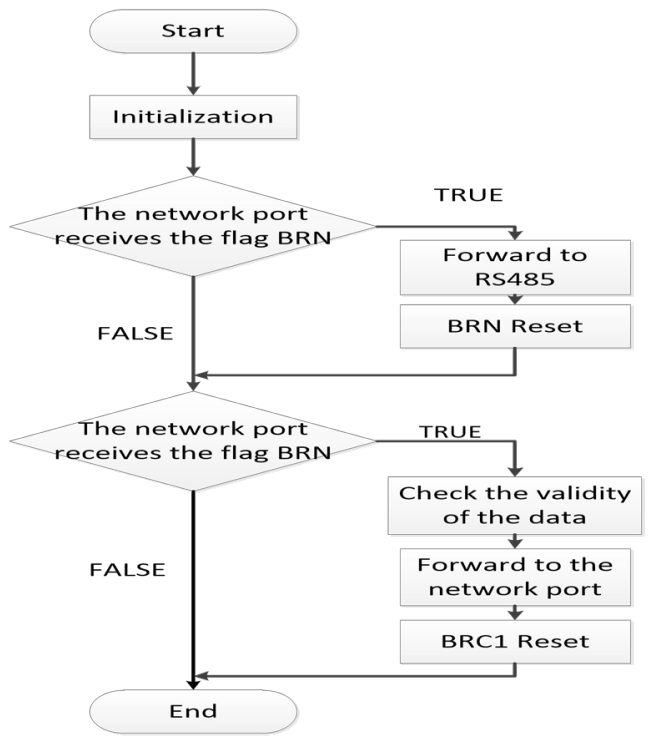

Figure 2. Collect the flow chart of the metering terminal

\subsection{Tap water flow meter data collection}

Because the tap water flow meter uses the third party product, its communication agreement uses the factory FE FE 6819 as the guide character customization agreement. The data collector uses the regular poll flow meter data, checks after assembling the data frame waiting to send, responds to the data center Patrol command to send data frames. Acquisition of three flow meter program shown in Figure 3.

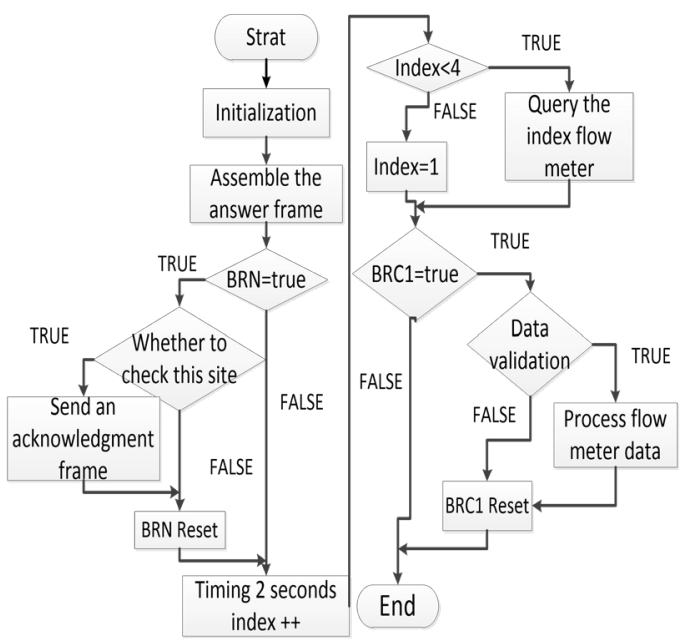

Figure 3. Flow chart of collecting flow meter program

\subsection{Central air conditioning data collection}

The integrated office building of the eastern of the Shandong Academy of Sciences used Central air-conditioning system, which reserved MODBUS data remote interface. The data collector collect central air-conditioning operation data in accordance with MODBUS agreement. The Central air-conditioning data acquisition process flow shown in Figure 4.

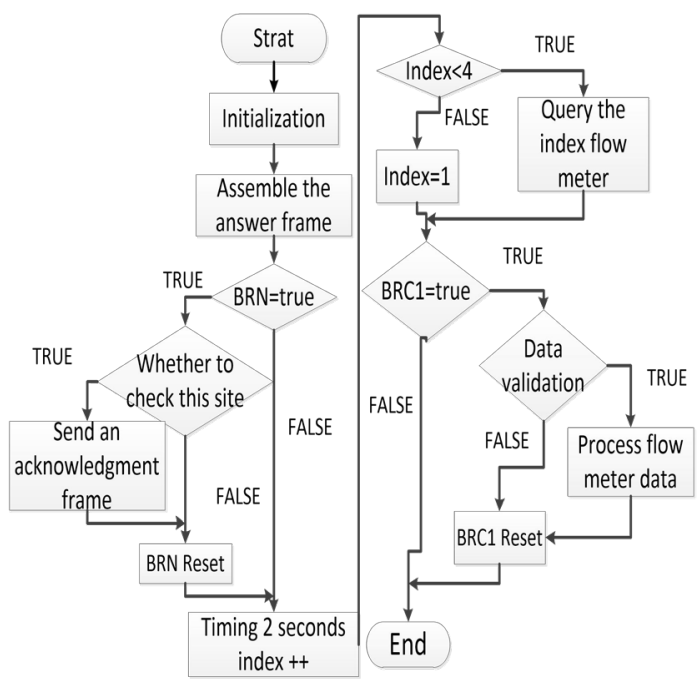

Figure 4. Flow chart of collecting central air conditioning program 


\section{Conclusions}

Through the operation of the past six months, the energy consumption monitoring and management system of the comprehensive office building of the eastern district of Shandong Academy of Sciences is stable and reliable. The relevant leadership and management departments can grasp the energy consumption of the buildings through real-time dynamic building energy monitoring. Query analysis of building energy consumption rules, energy consumption trends and other information to improve the level of energy consumption management. Before the implementation of the system, the total cost of energy consumption per year of the office building is about 2.2 million yuan. After the implementation of the system, the total cost of the system is reduced to 1.98 million yuan, which is achieved by saving $10 \%$ of the energy consumption. It has good economic benefits and social benefit.

\section{Acknowledgement}

This work has been supported by the project of the Shandong Province independent innovation and the results of special conversion (2014CGZH0804), Major Projects of Science and Technology in Shandong Province
(2015ZDZX10002,2015ZDXX0101G05) and Shandong Province key research and development plan (2017GGX30117) and Technology research(GG201703090037).

\section{References}

1. National Ministry of Housing and Urban and Rural Development. Technical Guidelines for Data Acquisition of Energy Consumption in State Office Building and Large Public Building Energy Consumption Monitoring System.S.

2. ChangChu Miao. The Programming Method and Engineering Application of Programmable Logic Controller. M. ChongQing: Chongqing University Press, 2001.

3. YunXin Gong, HouYu Zhao, Benzhi Qi Editor. PLC technology and application: based on Siemens57-200. Tsinghua University Press. 2009,9.

4. Feng Zhao, Guanghe Cheng, Xiang Sun, Xuan Luo, XuXai Ren. Journal of electronic measurement and instrument . 237-239, (2009).

5. HuiQiang Li, Guanghe Cheng, LingYan Han, KeYing Li, MaoLi Wang. Automation \& Instrumentation. 1, (2010), 17-20. 